PROCEEDINGS OF THE

AMERICAN MATHEMATICAL SOCIETY

Volume 97, Number 2, June 1986

\title{
THE EQUATIONS DEFINING A CURVE OF GENUS $4^{1}$
}

\author{
GEORGE R. KEMPF
}

\begin{abstract}
For most curves of genus 4 and characteristic $\geq 3$ the second osculating cone of the theta divisor is the cone over the canonical curve.
\end{abstract}

Let $C$ be a nonhyperelliptic complete smooth curve of genus 4 . The canonical morphism $C \rightarrow \mathbf{P}^{3}$ is an embedding and the image is defined as the intersection of a quadric and a cubic. In this paper we will discuss a geometric way of constructing such equations from the theta divisor on the Jacobian of $C$.

Recall that the singularities of the theta divisor correspond to linear systems $|D|$ on $C$ of dimension one and degree 3 . For a general curve there are two such systems $|D|$ and $|K-D|$. for some special curves there is only one $|D|=|K-D|$. We will give a construction of the equations of the canonical curve when the theta divisor has two singular points. This construction was suggested by the work of Ehrenpreis and Farkas $[\mathbf{1}]$ where they show that it works for an unknown but sufficiently general Riemann surface.

We will indicate the construction in the Riemann surface case. Let $\mathbf{C}^{4}$ be the universal covering space of the Jacobian. Let $x$ in $\mathbf{C}^{4}$ be a point corresponding to a $g_{3}^{1}$, say $|D|$, on $C$. We make the power series expansion $\theta(y)=f_{2}+f_{3}+\cdots$ of the theta function at $x$ where $f_{i}$ is a homogeneous polynomial of degree $i$ in $y-x$.

The polynomials $f_{2}$ and $f_{3}$ may be identified with forms on the canonical space $\mathbf{P}^{3}$. As such their zeroes contain the canonical curve. We can prove that if $|2 D| \neq$ $|K|, f_{2}$ and $f_{3}$ define the canonical curve. (If $|2 D|=K, f_{3}=0$ and the method fails.) We will formulate our results algebraically so that they hold for a curve over any field of characteristic not equal to two.

1. Flat varieties and osculating cones. We will be working with schemes of finite type over an algebraically closed field $k$. Points will always be $k$-rational. If $x$ is a point on a scheme $X$, for any positive integer $n$ the scheme $X_{n x}$ is the $n$th infinitesimal neighborhood of $x$. It is the closed subscheme of $X$ defined by the power ideal $I^{n+1}$ where $I$ is the ideal of $x$.

A flat structure of $n$th order on a smooth variety $X$ at the point $x$ is an isomorphism $i: T_{n 0} \stackrel{\approx}{\rightarrow} X_{n 0}$ where $T$ is a vector variety. In this situation $T$ may be identified with the tangent space of $X$ at $x$. We henceforth will fix such a flat structure. A smooth subvariety $Y$ of $X$ passing through $x$ is flat to the $n$th order if $i^{-1}\left(Y_{n x}\right)$ has the form $S_{n 0}$ where $S$ is a linear subspace of $T$. Here $S$ may be identified with the tangent space of $Y$ at $x$.

Let $X_{1}, \ldots, X_{n}$ be regular functions on $X$ at $x$ such that $i^{*} X, \ldots, i^{*} X_{n}$ are a basis for the linear functions on $T_{n 0}$. The $X_{j}$ are determined modulo $I^{n+1}$ by their

Received by the editors October 8, 1984 and, in revised form, June 25, 1985.

1980 Mathematics Subject Classification. Primary 14H40; Secondary 30F10.

${ }^{1}$ Partly supported by NSF grant \#75-05578. 
inverse images $i^{*} X_{j}$. Let $f$ be an element of $I^{n} / I^{m+n+1}$. Then we have a unique expansion $f \equiv f_{m}+\cdots+f_{m+n+1}\left(\left(I^{m+n}\right)\right)$ where the $f_{j}$ is a homogeneous polynomial of degree $j$ in the $X_{k}$ 's. By using $i^{*}$ we may regard the $f_{j}$ 's as homogeneous polynomials on $T$. We will call them the components of $f$ and they do not depend on the choice of the $X_{j}$.

Let $D \subset X$ be a divisor passing through $x$. Take any equation $f=0$ for $D$ at $x$. Let $m$ be the multiplicity of $D$ at $x$. Then $m$ is the integer such that $f \in I^{m}-I^{m+1}$. Define the $n$th osculating cone $O_{n}(D, x)$ to be the closed subscheme of $T$ defined by the equations $f_{m}=\cdots=f_{m+n+1}=0$ where the $f_{j}$ 's are the components of $f$. An obvious calculation shows that $O_{n}(D, x)$ is independent of the choice of the defining function $f$.

When $n=1$ the osculating cone $O_{1}(X, x)$ is just the tangent cone of $D$ at $x$. If we have compatible flat structures, $O_{j}(X, x) \supseteq O_{j+1}(X, x)$.

If $E$ is a flat curve through $x$, the tangent line to $E$ at $x$ is contained in $O_{n}(X, x)$ if and only if the function $f$ restricted to $E$ vanishes at least to the $(m+n)$ th order at $x$. Thus $E$ kisses $D$ to high order if and only if its direction is in the osculating cone.

We need an example. Let $X$ have a flat structure of order 2 at $x$. Let $\alpha=\left(\begin{array}{ll}a & b \\ c & d\end{array}\right)$ be a $2 \times 2$ matrix of regular functions on $X$ at $x$ such that the value of each coefficient is zero at $x$. Then the determinant $\operatorname{det} \alpha$ is a regular function on $X$ which is contained in $I^{2}$ where $I$ is the ideal of $x$. We have the expansion $\operatorname{det} \alpha \equiv(\operatorname{det} \alpha)_{2}+(\operatorname{det} \alpha)_{3}$ modulo $I^{4}$. This expansion can be computed from the expansion of the coefficients of $\alpha$; in fact, $(\operatorname{det} \alpha)_{2}=a_{1} d_{1}-b_{1} c_{1}$ and $(\operatorname{det} \alpha)_{3}=a_{1} d_{2}+a_{2} d_{1}-b_{1} c_{2}-b_{2} c_{1}$. For the "general" case where $(\operatorname{det} \alpha)_{2} \neq 0$, the $\operatorname{divisor} \operatorname{det} \alpha=0$ say $D$ has a double point at $x$ and its second osculating cone $O_{2}(D, x)$ is defined by the two equations $(\operatorname{det} \alpha)_{2}=(\operatorname{det} \alpha)_{3}=0$. We want to study when the osculating cone is smaller than the tangent cone $O_{1}(D, x)$ which is defined by the one equation $(\operatorname{det} \alpha)_{2}=0$.

If $E$ is a flat curve through $x$ with parameter function $\pi$ vanishing at $x$, the matrix $\left.\alpha\right|_{E}$ is equivalent to a matrix $\left(\begin{array}{cc}\pi^{n} & 0 \\ 0 & \pi^{m}\end{array}\right)$ where $1 \leq n \leq m \leq \infty$. Thus $\left.(\operatorname{det} \alpha)\right|_{E}=$ (unit at $\left.x\right) \pi^{n+m}$. Hence the direction of $E$ is contained in $O_{i}(D, x)$ if and only if $n+m \geq 2+i$. In particular, the direction of $E$ is contained in the complement $O_{1}(D, x)-O_{2}(D, x)$ if and only if $n=1$ and $m=2$. We may interpret the numbers $n$ and $m$ in terms of the $\operatorname{ker}\left(\left.\alpha\right|_{E_{p x}}\right)$ where $p$ is a nonnegative integer. If $p^{\prime} \geq p$ we have an obvious homomorphism $R_{p^{\prime}, p}=\operatorname{ker}\left(\left.\alpha\right|_{E_{p^{\prime} x}}\right) \rightarrow \operatorname{ker}\left(\left.\alpha\right|_{E_{p x}}\right)$. The integer $n$ is the minimum $p$ such that $R_{p, 0}$ is not surjective and $m$ is the minimum $p$ such that $R_{p, 0}$ is zero. Thus to obtain a direction in $O_{1}(D, x)-O_{2}(D, x)$ we need to have $R_{1,0}$ not surjective and nonzero but $R_{2,0}$ zero.

Next we will make an assumption. Consider the closed subscheme $Y$ in $X \times \mathbf{P}^{1}$ defined by the equations $a S_{0}+c S_{1}=b S_{0}+d S_{1}=0$ where $S_{0}$ and $S_{1}$ are basic homogeneous one-forms on $\mathbf{P}^{1}$. We will assume that $Y$ is a smooth variety of dimension equal to $\operatorname{dim} X-1$. By general reasoning found in [2 or 3], the projection onto the factor $X$ maps $Y$ birationally onto the divisor $D$. Furthermore, the normal bundle $N$ of $Y$ along $x \times \mathbf{P}^{1}$ is defined by the equations $a_{1} S_{0}+c_{1} S_{1}=b_{1} S_{0}+d_{1} S_{1}=$ 0 in $T \times \mathbf{P}^{1}$, and it is smooth of dimension equal to $\operatorname{dim} X-1$. Similarly, projection onto the factor $T$ maps $N$ birationally onto the tangent cone $O_{1}(D, x)$. Another general fact is that the morphism $N \rightarrow O_{1}(D, x)$ is birational exactly over the smooth part of $O_{1}(D, x)$. 
The above results imply that $D$ and $O_{1}(D, x)$ are reduced. Also, $O_{1}(D, x)$ is a rank 3 or 4 quadric with singular locus $S=\left\{a_{1}=b_{1}=c_{1}=d_{1}=0\right\}$ which is properly contained in $O_{1}(D, x)$. Furthermore, for a curve $E$ as above, the number $n$ equals 1 if and only if its direction lies in $O_{1}(D, x)-S$. Here one should also note that $S$ is contained in $O_{2}(D, x)$ by looking at their equations. Finally, to find a direction $J$ of $E$ in $O_{1}(D, x)-O_{2}(D, x)$ we need to have (a) $J$ in $O_{1}(D, x)-S$, and (b) any element of $\operatorname{Ker}\left(\left.\alpha\right|_{E_{1 x}}\right)$ is not in the image of $R_{2,1}$.

2. The flat structure on groups and the Picard scheme. Let $G$ be an algebraic group. We will define a flat structure on $G$ at the identity which may be translated either right or left to give flat structures at any point of $G$. The special role of the identity is that it is the fixed point for the commuting family $\{[n]\}_{n \in \mathbf{Z}}$ where $[n]$ is the $n$-power operation sending $g$ to $g^{n}$. The mapping tangent to $[n]$ at the identity is just multiplication by $n$. Consequently, if $I$ is the ideal of the identity $e,[n]^{*}$ acts on $I^{i} / I^{j+1}$ by multiplication by $n^{j}$.

The idea is to have our flat structure $T_{q 0} \rightarrow G_{q e}$ be equivariant so that multiplication by $n$ on $T_{q 0}$ is the same as the action of $[n]$ on $G_{q e}$. There is a unique such flat structure of order $q$ if the eigenfunctions $n \mapsto n^{j}$ for $0 \leq j \leq q$ are all distinct by elementary eigenspace considerations on $O_{g_{1} e} / I^{q+1}$. In fact, in this case we have $I^{j} / I^{q+1}=\bigoplus_{j \leq i \leq q} V_{i}$, where $V_{i}$ is the $n^{i}$-eigenspace in $O_{g, e} / I^{q+1}$. In characteristic zero this happens for all possible choices of $q$. In fact, these flat structures in the limit give the analytic isomorphism between a neighborhood of zero in $T$ and a neighborhood of the identity in $G$, which is called canonical coordinates. In this case the isomorphism is called the exponential mapping. If the characteristic is a prime $p$, then if $q \leq p-1$, the eigenvalues are again all distinct as $\left(\mathbf{Z} /{ }_{p} \mathbf{Z}\right)^{*}$ is cyclic and $p \not \equiv p^{0}$ modulo $p$. Thus we have a canonical flat structure of order $p-1$ on $G$ at $e$ in characteristic $p$. In summary, we have a canonical flat structure of order $q$ on $G$ at $e$ if $q$ is a nonnegative integer if the characteristic is zero, or if $q$ is strictly less than $p$ if the characteristic is a prime $p$.

Let $E$ be a smooth curve in $G$ passing through $e$. Then $E$ is flat for our canonical flat structure of order $q$ if and only if $[n] E_{q \cdot e} \subset E_{q \cdot e}$ for all integers $n$. Evidently this condition is necessary because $n H \subset H$ for any vector subspace $H$ of $T$. To see that it is sufficient just consider the eigenspace decomposition of the invariant ideal of $E_{q \cdot e}$ in $G_{g \cdot e}$ and you should find that it is generated by $n$-eigenvectors. This shows that $E$ is flat at $q$.

Let $C$ be a smooth complete curve of arbitrary genus. Then we have the Picard scheme $\operatorname{Pic}(C)$ which parameterizes isomorphism classes of invertible sheaves on $C$. In other words, $\operatorname{Pic}(C)=H^{1}\left(C, O_{C}^{*}\right)$. Thus $\mathrm{Pic}(C)$ has a natural abelian algebraic group structure corresponding to the tensor product of sheaves. The identity of $\mathrm{Pic}(C)$ is denoted by 0 and corresponds to the trivial sheaf $O_{C}$.

Let $q$ as before be an integer with $0 \leq q$ and $q<$ characteristic if prime. Let $X_{q}$ be the infinitesimal scheme $\operatorname{Spec}\left(k[X] /\left(X^{q+1}\right)\right)$ with one point, say $x_{0}$. The assignment

$$
a \mapsto 1+a X+\frac{a^{2} X^{2}}{2 !}+\cdots+\frac{a^{q} X^{q}}{q !}=\exp _{q}(a X)
$$

defines a sheaf homomorphism from $O_{C}$ to the sheaf of units on $C \times X_{q}$ which are the identity on $C \times x_{0}$. This assignment induces a mapping of first cohomology which sends an element $\eta$ of $H^{1}\left(C, O_{C}\right)$ to a morphism $\exp _{q}(\eta): X_{q} \rightarrow \operatorname{Pic}(C)$ such that 
$\exp _{q}(\eta)\left(x_{0}\right)=0$. When $q=1$, this gives the usual identification between $H^{1}\left(C, O_{C}\right)$ and the tangent space to $\operatorname{Pic}(C)$ at 0 where $\eta$ corresponds to the tangent vector $T\left(\exp _{q}(\eta)\right)(d / d X)$. For general $q$, we have $\left(\exp _{q}(\eta)\right)^{n}=\exp (n \eta)$ for all integers $n$. Hence if $\eta \neq 0, \exp _{q}(\eta)$ is a closed immersion and its image has the form $E_{q 0}$ where $E$ is a flat curve local around 0 in the canonical flat structure on the group $\operatorname{Pic}(C)$.

We want to give an explicit description of the invertible sheaf $L_{\eta, q}$ on $C \times X_{q}$ whose classifying morphism is $\exp _{q}(\eta)$ for some element $\eta$ of $H^{1}\left(C, O_{C}\right)$. Let $U$ be an open subset of $C$ with complement $F$. Let $f$ be a rational function on $C$. We will define a subsheaf $L_{f, F, q}$ of $\operatorname{Rat}(C) \otimes_{k} k[X] /\left(X^{q+1}\right) \supseteq \mathcal{O}_{C \times X_{q}}$. A section of $L_{f, F, q}$ is a series $a=a_{0}+a, X+\cdots+a_{q} X^{q}$ where the $a_{i}$ 's are rational functions on $C$ such that $a$ is regular at points in $U$ and $\exp _{q}(f) \cdot a$ is regular at points in $F$. Then $L_{f, F, q}$ is a free $O_{C \times X_{q}}$-module with base 1 over $U$ and $\exp _{q}(-f)$ over a small neighborhood of $F$. By the functional equation for $\exp _{q}$, $L_{f, F, q}$ depends only on the polar behavior of $f$ at each point of $F$. In other words, $L_{f, F, q}=L_{p p_{F}(f), q}$ where $p p_{F}(f)$ denotes the sum of the principal parts of $f$ at the points of $F$. Furthermore, for a rational function $g$ on $d$, multiplication by $\exp _{q}(g)$ maps $L_{\omega, q}$ onto $L_{\omega+(g), q}$ where $(g)$ denotes the total principal part of $g$. Thus the isomorphism class of $L_{\omega, q}$ depends on the cohomology class $[\omega]=\eta$ of $\omega$ in $\operatorname{Prin}\left(O_{C}\right) / \operatorname{Im} \operatorname{Rat}(C)=H^{1}\left(C, O_{C}\right)$. In our case $L_{\omega, q}$ is a representative of the isomorphism class $\mathcal{L}_{\eta, q}$. Lastly, for $i<q$ we have $L_{\omega, q} \mid C \times X_{i}=L_{\omega, i}$, an exact sequence $0 \rightarrow O_{C} X^{i+1} \rightarrow L_{\omega, i+1} \rightarrow L_{\omega, i} \rightarrow 0$ and $L_{\omega, 0}=O_{C}$. Furthermore, the cohomology class of the extension $0 \rightarrow O_{C} X \rightarrow L_{\omega, 1} \rightarrow L_{\omega, 0} \rightarrow 0$ is just $\eta \cdot X$.

3. The osculating cone to the theta divisor. Let $g$ be the genus of our curve $C$. Let $P \equiv \mathrm{Pic}_{g-1}(C)$ be the component of the Picard scheme of $C$ which parameterizes sheaves of degree $g-1$. Then we have an irreducible divisor $\theta$ on $P$ whose points represent sheaves $\mathcal{N}$ where $\Gamma(C, \mathcal{N}) \neq 0$. One may find references to the general theory of the theta divisor in [2 and 3].

Let $\mathcal{M}$ be an invertible sheaf on $C$ of degree $g-1$ with $\operatorname{dim} \Gamma(C, \mathcal{M})=2$. Let $m$ be the corresponding point of $P$. Then $m$ is a double point of the theta divisor. We may find a neighborhood $\mathcal{V}$ of $m$ and a matrix $\alpha=\left(\begin{array}{ll}a & b \\ c & d\end{array}\right)$ of regular functions on $\mathcal{V}$ such that $\alpha$ is an approximating homomorphism for computing the variation of the cohomology in a universal family $\mathcal{N}$ of invertible sheaves on $C$ parameterized by $P$. In particular, for any morphism $f: S \rightarrow P$ from an affine scheme $S$ which factors through $\mathcal{V}, \operatorname{Ker}\left(f^{*} \alpha\right)$ is naturally isomorphic to $\Gamma\left(C \times S,\left(1_{C} \times f\right)^{*} \mathcal{N}\right)$ where $\left(1_{C} \times f\right)^{*} \mathcal{N}$ is a particular family of sheaves on $C$ parameterized by $S$.

In this situation our assumption on $\alpha$ of $\S 1$ holds and the $\operatorname{divisor} \operatorname{det}(\alpha)=0$ is $\theta \cap \mathcal{V}$ and each coefficient of $\alpha$ vanishes at $m$. So we may apply the previous theory to study the theta divisor at the point $m$. First we will describe some of the rich geometry of the tangent cone $O_{1}(\theta, m)$ which is contained in the tangent space of $P$ at $m$ that is naturally isomorphic to $H^{1}\left(C, O_{C}\right)$. As we have seen $O_{1}(\theta, m)$ is a quadric of rank 3 or 4 . A vector $\eta$ in $H^{1}\left(C, O_{C}\right)$ is contained in $O_{1}(\theta, m)$ if and only if the cup product $\bigcup \eta: \Gamma(C, \mathcal{M}) \rightarrow H^{1}(C, \mathcal{M})$ is not surjective. The singular locus $S$ of $O_{1}(\theta, m)$ consists of those $\eta$ with $\bigcup \eta=0$ and is a linear space of codimension 3 or 4 . Geometrically $O_{1}(\theta, m)$ is the image of a $g-2$ rank vector bundle over $\mathbf{P}^{1}$. In terms of the cup product this is given as follows: Let $\alpha$ be a nonzero element of $\Gamma(C, \mathcal{M})$, let $K(\alpha)$ be the kernel of the surjection $\bigcup \alpha: H^{1}\left(C, O_{C}\right) \rightarrow H^{1}(C, \mathcal{M})$. 
Then $K(\alpha)$ only depends on the line generated by $\alpha$ and we have $O_{1}(\theta, m)=$ $\bigcup_{(\alpha) \in \operatorname{Lines}(\Gamma(C, \mathcal{M}))} K(\alpha)$. Note also that the codimension two subspaces $K(\alpha)$ are not contained in $S$.

We can analyze the space $K(\alpha)$ more completely. Take an isomorphism $\mathcal{M} \underset{\rightarrow}{\approx}$ $O_{C}(D)$ where $D$ is the divisor of zeroes of $\alpha$ such that $\alpha$ corresponds to 1 . By the long exact sequence of $\left.0 \rightarrow O_{C} \rightarrow O_{C}(D) \rightarrow O_{C}(D)\right|_{D} \rightarrow 0$ we have an exact sequence

$$
0 \rightarrow k \rightarrow \Gamma\left(C, O_{C}(D)\right) \rightarrow \Gamma\left(C,\left.O_{C}(D)\right|_{D}\right) \rightarrow K(\alpha) \rightarrow 0 .
$$

Thus the cohomology classes $K(\alpha)$ are given by principal parts bounded by $D$ modulo the total principal parts of sections on $O_{C}(D)$. Recall $[4]$ that the canonical embedding of $C$ as lines in $H^{1}\left(C, O_{C}\right)$ sends a point $c$ to the line generated by the class of a principal part $\omega_{c}$ with its only singularity being a pole of order one at $c$. The set $\tilde{C}=0 \coprod\left\{\right.$ all $\omega_{c}$ is the cone in $H^{1}\left(C, O_{C}\right)$ over the canonical curve $\}$. As the linear system $|D|$ moves, we may find some $\alpha$ such that $D$ contains any given point $c$ of $C$. Then the cohomology class of $\omega_{c}$ is contained in $K(\alpha)$ and, hence, $\tilde{C} \subset \mathrm{O}_{1}(\theta, m)$.

The last fact from the general theory that we will recall involves the sections of a first order infinitesimal deformation of the sheaf $\mathcal{M}$. Let $\eta=[\omega]$ be the cohomology class of a principal part $\omega$ of a rational function. Then $\eta$ determines a curve $f: X_{1} \rightarrow P$ such that $f\left(x_{0}\right)=m$ with velocity $T(f)(d / d x)=\eta$. The sheaf $f^{*} \mathcal{L}$ is isomorphic to $L_{\omega, 1} \otimes \mathcal{M}$. The restriction $R_{1,0}: \operatorname{Ker}\left(f^{*} \alpha\right) \rightarrow \operatorname{Ker}(\alpha(m))$ is isomorphic to the restriction $T_{1,0}: \Gamma\left(C \times X_{1}, L_{\omega, 1} \otimes \mathcal{M}\right) \rightarrow \Gamma(C, \mathcal{M})=\Gamma\left(C, L_{\omega, 0} \otimes \mathcal{M}\right)$. In this situation Image $\left(T_{1,0}\right)=\operatorname{Kernel}\left(\bigcup \eta: \Gamma(C, \mathcal{M}) \rightarrow H^{1}(C, \mathcal{M})\right)$. In particular, $\eta$ is in $O_{1}(\theta, m)$ if and only if $T_{1,0}$ is not zero. Also, $\eta$ is in $S$ if and only if $T_{1,0}$ is surjective.

Now we will assume that the characteristic is not two. Then $q=2$ satisfies the previous conditions and we have a second order flat structure on $P$ at $m$. Consider the family $L_{\omega, 2} \otimes \mathcal{M}$ of sheaves on $C$ parameterized by $X_{2}$. This family has a classifying morphism $q: X_{2} \rightarrow P$ such that $\left.g\right|_{X_{1}}=f$ and $g$ is a closed immersion if $\eta \neq 0$. In this case, $g\left(X_{2}\right)$ is the second order expansion of a flat curve at $m$, and we may apply the previous theory to the restriction $R_{2,1}$. As before, the restriction $R_{2,1}: \operatorname{Ker}\left(g^{*} \alpha\right) \rightarrow \operatorname{Ker}\left(f^{*} \alpha\right)$ is isomorphic to the restriction $T_{2,1}: \Gamma\left(C \times X_{2,} L_{\omega, 2} \otimes \mathcal{M}\right) \rightarrow \Gamma\left(C \times X_{1,} L_{\omega, 1} \otimes \mathcal{M}\right)$.

The relevant results that we have so far are summarized as follows:

(1) $\eta \in \mathcal{O}_{1}(\theta, m)-S \leftrightarrow \eta \in K(\alpha)-S$ for some element $\alpha$ of $\Gamma(C, \mathcal{M})$.

Assume that (a) is satisfied. Then

(b) the image of $T_{1,0}$ is a line, and

(c) $\eta \in \mathrm{O}_{2}(\theta, m)$ if and only if Image $\left(T_{2,1}\right)$ does not contain any element with nonzero image under $T_{1,0}$.

4. Curves of genus four. We will now assume that our curve $C^{1}$ has genus four and is nonhyperelliptic. Then $\mathcal{M}$ corresponds to a $g_{3}^{1}=|D|$ which has no base points as $C$ is nonhyperelliptic. Furthermore, the corresponding morphism of degree $3 C \rightarrow P^{1}$ is necessarily separable as $C$ is not rational in characteristic three. Thus a general member of the linear system $|D|$ consists of three distinct points. In other words, almost all sections $\alpha$ of $\mathcal{M}$ have three distinct zeroes, say $a, b$ and $c$, where $D=a+b+c$. If we take an isomorphism $\mathcal{M} \approx O_{C}(D)$ as before, there is a rational function $\beta$ in $\Gamma\left(C, O_{C}(D)\right)$ with poles of order one at exactly 
$a, b$ and $c$. Let $\omega_{*}$ be the principal part of $\beta$ at $*$ in $\{a, b, c\}$. In this case, $K(\alpha)$ is spanned by $\omega_{a}, \omega_{b}, \omega_{c}$ modulo the relation $\omega_{a}+\omega_{b}+\omega_{c}=0$. Thus the vector space $K(\alpha)$ has basis $\left[\omega_{a}\right]$ and $\left[\omega_{b}\right]$.

Let $\omega=\lambda_{a} \omega_{a}+\lambda_{b} \omega_{b}$ for some constants $\lambda_{a}$ and $\lambda_{b}$. Let $\eta=[\omega]$. Let us consider the sequence of sheaves

$$
0 \rightarrow X O_{C}(D) \rightarrow L_{\omega, 1} \otimes O_{C}(D) \rightarrow O_{C}(D) \rightarrow 0 .
$$

Then $\alpha=1$ lifts to the section 1 of $L_{\omega, 1} \otimes_{k} O_{C}(D)$ and $\beta$ lifts to a section of $L_{\omega, 1} \otimes_{k} O_{C}(D)$ if and only if $\beta \cdot \omega=0$ as a cohomology class in $H^{1}\left(C, O_{C}(D)\right)$ if and only if $\eta \in S$. To understand the singular set $S$ there are two cases:

(A) $\mathcal{M} \not \Omega_{C} \otimes \mathcal{M}^{\otimes-1}$, and

(B) $\mathcal{M} \approx \Omega_{C} \otimes \mathcal{M}^{\otimes-1}$.

In case (A), $S=0$ and $O_{1}(\theta, m)$ is a rank four quadric. In case (B), $S$ is a line and $O_{1}(\theta, m)$ is of rank three. To see this, we need to compute the image of the cup product $\bigcup: H^{1}\left(C, O_{C}\right) \rightarrow \operatorname{Hom}\left(H^{0}(C, \mathcal{M}), H^{1}(C, \mathcal{M})\right)$. The kernel of $\bigcup$ is dual to the image of the multiplication $z: H^{0}(C, \mathcal{M}) \otimes H^{0}\left(C, \Omega_{C} \otimes \mathcal{M}^{\otimes-1}\right) \rightarrow H^{0}\left(C, \Omega_{C}\right)$. By the base point free pencil trick, $\operatorname{rank}(z)=4$ in case (A) and 3 in case (B). Anyway we may assume that $\eta \notin S$. In this case $k \cdot 1$ is the image of the restriction $T_{1,0}$. Thus, we need to examine when $1+(\gamma+\delta \beta) X$ is in the image of the restriction $T_{2,1}$ for some choices of constants $\gamma$ and $\delta$ to determine whether $\eta$ is contained in $\mathrm{O}_{2}(\theta, m)$ or not.

Thus we have to examine when we have a section of $L_{\omega, 2} \otimes_{k} O_{C}(D)$ of the form $1+(\gamma+\delta \beta) X+j X^{2}$ where $j$ is a rational function on $C$. Here $j$ is a regular section of $O_{C}(D)$ over $C-\{a, b\}$. The condition on $j$ at the points $a$ and $b$ is more complicated. We need

$$
\begin{aligned}
(1+\omega X & \left.+\omega^{2} X^{2} / 2\right)\left(1+(\gamma+\delta \beta) X+j X^{2}\right) \\
& =1+\omega+(\gamma+\delta \beta) X+\left(\omega^{2} / 2+\omega(\gamma+\delta \beta)+j\right) X^{2}
\end{aligned}
$$

to be regular as a section of $O_{C}(D) \otimes_{R} O_{X_{2}}$ at the points $a$ and $b$. In particular, $j$ is a section of $O_{C}(D+a+b)$ as $\omega$ is bounded by $D$.

Assume that we are in case (A). Then $|2 D| \neq|K|$ and $\operatorname{det}(2 D)=6$. Thus $\operatorname{dim} \Gamma\left(C, O_{C}(2 D)\right)=3$ and it has basis $1, \beta, \beta^{2}$. Hence, $\Gamma\left(C, O_{C}(D+a+b)\right)=$ $\Gamma\left(C, O_{C}(D)\right)$. Thus $j=T_{1}+T_{2} \beta$ where the $T_{i}$ 's are constants. In this case the conditions become that $\lambda_{*}^{2} \beta^{2} / 2+\lambda_{*} \beta(\gamma+\delta \beta)+T_{1}+T_{2} \beta$ is a regular section of $O_{C}(D)$ at $*=a$ and $b$. In other words, we need $\lambda_{*}\left(\lambda_{*} / 2+\delta\right)=0$ for $*=a$ and $b$. Thus if $\lambda_{a}=0$ or $\lambda_{b}=0$, we may always find $J$ satisfying the equations. If $\lambda_{a} \neq 0 \neq \lambda_{b}$, then $\delta=-\lambda_{a} / 2=-\lambda_{b} / 2$ and we have a solution if and only if $\lambda_{a}=\lambda_{b}$. In conclusion, $\eta \subset O_{2}(\theta, m)$ if and only if $\eta$ is on a line spanned by $\left[\omega_{a}\right],\left[\omega_{b}\right]$ or $\left[\omega_{c}\right]$.

Thus we have shown two things. The osculating cone $O_{2}(\theta, m)$ is properly contained in the tangent cone $O_{1}(\theta, m)$ and the cone over the canonical curve $\tilde{C}$ is contained in $O_{2}(\theta, m)$ when $(A)$ holds. In this case because $O_{2}(\theta, m)$ is defined by a quadric and a cubic in the four dimension $H^{1}\left(C, O_{C}\right)$ and $C$ has genus four, we may conclude that $\tilde{C}=\mathrm{O}_{2}(\theta, m)$.

THEOREM. If $\mathcal{M} \not \Omega_{C} \otimes \mathcal{M}^{\otimes-1}$, then $\tilde{C}=\mathrm{O}_{2}(\theta, m)$. 
As an additional fact in case (B), we have

LEMMA. If $\mathcal{M} \approx \Omega_{C} \otimes \mathcal{M}^{\otimes-1}$, then $O_{1}(\theta, m)=O_{2}(\theta, m)$.

This lemma can be proven by the previous argument but it is even easier. Let $i(n)=k-n$ be the usual involution of $P$ where $k=\left[\Omega_{C}\right]$. In case $(\mathrm{B}), m$ is a fixed point of $i$ and $\theta$ is invariant under $i$. Let $f=0$ be an equation for $\theta$ at $m$. Then $f(i(x))=u(x) \cdot f(x)$ where $u(m)=1$. Expanding modulo $I^{3}$, we have $f_{2}-f_{3}=\left(1+u_{1}\right)\left(f_{2}+f_{3}\right)$ and hence $f_{3}=-\frac{1}{2} u f_{2}$. Therefore, the scheme $O_{2}(\theta, m)=\left\{f_{2}=f_{3}=0\right\}$ equals $O_{1}(\theta, m)=\left\{f_{2}=0\right\}$.

\section{REFERENCES}

1. L. Ehrenpreis and H. M. Farkas, Some refinements of the Poincaré period relation, Discontinuous Groups and Riemann Surfaces, Ann. of Math. Stud., No. 79, Princeton Univ. Press, Princeton, N. J., 1974, pp. 105-120.

2. G. Kempf, On the geometry of a theorem of Riemann, Ann. of Math. (2) 98 (1973), 178-185.

3. __ Abelian integrals, Monografias Inst. Mat. No. 13, Univ. Nacional Autonoma Mexico, 1984.

4. __ On algebraic curves, Crelle's J. 295 (1977), 40-48.

Department of Mathematics, Johns Hopkins University, Baltimore, MaRYLAND 21218 